\title{
La Nouvelle Manga et le cinéma ${ }^{1}$
}

\section{Temenuga Trifonova York University}

(Traduction de Chris Reyns-Chikuma et Sylvain Rheault)

La Nouvelle Manga (NM) est un mouvement artistique qui réunit la narration cinématique de la bande dessinée franco-belge (BD) ${ }^{2}$ avec les histoires quotidiennes des mangas japonais. ${ }^{3}$ Le terme a d'abord été utilisé par Kiyoshi Kusumi, éditeur du magazine manga Comickers, pour décrire les romans graphiques de Frédéric Boilet perçus par les Japonais comme proches d'un point de vue graphique de la BD française, mais qui se lisent comme des mangas et sont imprégnés par l'esprit du cinéma et de la littérature français. Alors que les comics traditionnels américains et la BD française mettaient l'accent sur l'art graphique, la NM utilise une narration cinématique, cadre par cadre pour raconter des histoires s’inspirant du quotidien. Parmi les artistes associés avec le mouvement, on compte Frédéric Boilet, Nicolas de Crécy, Jiro Taniguchi, Kan Takahama, Kazuichi Hanawa, Kiriko Nananan, Hideji Oda, Yoshiharu Tsuge, et Naito Yamada.

La nature cinématique de la NM a été perçue comme un exemple de ce que Bart Beaty voit comme « the visual turn » (le tournant visuel) dans les comics, depuis le début des années 1990, s'éloignant du « littéraire » et se tournant vers les arts visuels (en particulier, le cinéma). Bien qu'on puisse facilement retracer le caractère cinématique de la NM jusqu'à la Nouvelle Vague française, dans ce qui suit je soutiens que ce qui rend la NM cinématique n'est pas seulement, ou pas nécessairement, l'usage particulier de techniques cinématiques, mais plutôt l'adoption d'un type particulier de narration propres à la Nouvelle Vague et au cinéma japonais.

Dans son manifeste de la NM, Boilet note qu'il y a actuellement trois marchés majeurs de la narration graphique — le Japon, les États-Unis et la

\footnotetext{
${ }^{1}$ Cet article a originellement été publié en anglais sous le titre « Nouvelle manga and cinema » dans Studies in Comics, August 2012, vol. 3 no1 pp. 47-62.

${ }^{2}$ Les définitions de la BD sont multiples et étendues. Voir entre autres, Grove; Carrier.

${ }^{3}$ Les mangas résultent du métissage de la tradition japonaise de l'imprimé avec le style caricatural des magazines humoristiques comme Punch en Angleterre ou les strips des journaux américains. Les mangas japonais diffèrent des comics anglo-américains sur plusieurs aspects: 1) un rythme plus lent; 2) une plus grande variété de mangas; 3) un nombre réduit de cases par page et moins de récitatifs.
} 
France — représentés par trois « genres » distincts ou « styles nationaux »: manga, comics, et BD. Cependant, selon Boilet, cette classification ne s'applique pas à d'autres médias. Ainsi le cinéma, la littérature, et les arts visuels sont-ils tous considérés comme des formes artistiques universelles. C'est seulement dans la narration graphique que la classification s'appuie entièrement sur une comparaison des moyens de production propres aux mangas, aux comics et à la BD. Dès que nous laissons de côté les produits purement commerciaux de ces trois styles nationaux pour nous concentrer sur les narrations graphiques d'auteur — narrations sur la vie quotidienne destinées à des adultes —, il devient évident, selon Boilet, que les similarités entre les trois styles supposément distincts dépassent de loin les différences qui pourraient exister entre elles. C'est pourquoi il propose le terme «La Nouvelle Manga » pour désigner les œuvres qui s'adressent à un public plus vaste et plus divers, plutôt que de se limiter aux admirateurs de mangas ou de comics (ces admirateurs étant majoritairement des adolescents).

Si le cinéma français (et, plus largement, européen) s'est longtemps distingué du cinéma hollywoodien par l'attention portée à la vie de tous les jours, jusqu'au début des années 1990, la BD française ne s'est pas intéressée aux histoires inspirées de la vie ordinaire au quotidien, s'attachant plutôt à l'aspect esthétique. Contrairement aux bédéistes français, qui sont le plus souvent des illustrateurs, un mangaka (artiste manga) est d'abord un raconteur d'histoire. Plutôt que de ressasser les mêmes histoires d'aventures ou de science-fiction, un mangaka écrit des histoires enracinées dans la vie quotidienne, ce qui explique leur attrait pour un large éventail de lecteurs et lectrices, pas juste pour des «otaku» (fans de manga) mais pour toute personne qui lirait des bandes dessinées comme elle lit de la littérature ou va au cinéma. Selon la vision que se fait Boilet de la NM, l'engagement traditionnel du manga pour le quotidien et l'ordinaire a le potentiel de contrebalancer la nature de plus en plus hollywoodienne de la $\mathrm{BD}$ franco-belge, qui s'est cantonnée dans des genres établis comme la science-fiction, le film d'action, le film historique, ou le western. Des adaptations récentes de BD françaises en films illustrent parfaitement l'argument de Boilet. Immortal (2004), film de science-fiction français en langue anglaise, adapté de l'album La Foire aux Immortels d'Enki Bilal, est un des premiers longs métrages tournés entièrement avec un arrière-plan numérique, mêlant de vrais acteurs avec des décors générés par des ordinateurs. Les Chevaliers du ciel (2005), sorte de Top Gun à la française, adapté de la BD Tanguy et Laverdure de Jean-Michel Charlier et Albert Uderzo, raconte l'histoire de deux pilotes déjouant une attaque terroriste le jour des célébrations du 14 juillet à Paris. Largo Winch (2008), adapté de la BD du même titre, aspire clairement à devenir une franchise à la James Bond. Blueberry (2004), adapté de la BD de 
Giraud, vendu sur DVD sous le titre Renegade, est mis en marché comme un western conventionnel.

La NM s'inscrit dans l'héritage de la Nouvelle Vague et de Dogme, en appliquant leurs principes au monde de la BD. La Nouvelle Vague se caractérisait par une préoccupation en ce qui concerne l'authenticité : les réalisateurs et les réalisatrices approchaient la fiction comme si c'était un documentaire et le documentaire comme si c'était une fiction. De la sorte, ils évitaient tout ce qui fait artificiel ou scénarisé. Leurs films étaient guidés par deux tendances opposées en apparence: une prédilection pour la vérité, la spontanéité et l'improvisation et, en même temps, une tendance à l'autoréflexion. Leur conception de l'intrigue et des personnages était fortement influencée par le Nouveau Roman (Alain Robbe-Grillet, Georges Perec, Nathalie Sarraute, Michel Butor), qui déconstruisait l'artifice de l'intrigue linéaire et du personnage «tridimensionnel », l'illusion du point de vue omniscient, ainsi que les limites imprécises séparant la fiction de la réalité. Délaissant les normes du « cinéma de qualité » considéré comme stérile - des drames historiques parfaits du point de vue technique et des adaptations d'œuvres littéraires — les réalisateurs de la Nouvelle Vague ont fait de la sincérité et de l'intimité le nouveau standard de films à caractère personnel. De plus, ils ont cherché à légitimer le cinéma comme art indépendant de la littérature. La raison principale de leur insatisfaction envers le "cinéma de qualité » était précisément sa dépendance excessive à l'égard du scénario. Dans La Caméra-stylo, Alexandre Astruc a utilisé la métaphore de la « caméra-stylo » pour affirmer que le cinéma est un langage visuel que l'artiste peut utiliser pour exprimer des pensées et des sentiments autant abstraits que concrets.

Entre 1960 et 1980, la BD traditionnelle est passée par un processus de modernisation sous l'influence du Nouveau Roman et de la Nouvelle Vague. Elle a commencé à se distancer de la narration conventionnelle ${ }^{4}$ et à privilégier l'image indépendante sur la narration organisée en moments, ce qui résulta en une ambiguïté évocatrice plus efficace. ${ }^{5}$ L'influence de la Nouvelle Vague n'était

${ }^{4} \mathrm{Un}$ autre pont existe entre la $\mathrm{BD}$ et la littérature non-conventionnelles par l'entremise de l'Ouvroir de Bande Dessinée Potentielle (OuBaPo), inspirée de l'OuLiPo (Ouvroir de littérature potentielle) de Raymond Queneau et George Perec. Comme l'explique Grove, Within OuBaPo there are two categories: generatrices, works that create meaning, and transformatrices, works that transform meaning. In the first category are BDs that can be read upside down, back to front, via the folding of the page or in multiple directions; in the second, works featuring the implantation of cases by other authors or the removal of certain cases from the work [e.g. the removal of the letter 'e' from Perec's La Disparition]. (161)

${ }^{5}$ Même si elle utilise les techniques cinématographiques (angles de caméra, points de vue subjectifs, montage), la BD se distingue du cinéma de plusieurs façons: elle consiste en images 
que la première phase dans ce que les critiques ont décrit comme un « tournant visuel » dans l'histoire de la BD, qui a atteint son apogée dans les années 1990 au moment où les bédéistes commencèrent à s'inspirer des arts visuels et plastiques plutôt que de la littérature. L'apparition de petites maisons d'édition basée sur le modèle des coopératives d'artistes et le combat pour l'autonomie créative ont joué un rôle important dans la distanciation face aux traditions des écoles d'illustration franco-belge et dans l'émergence de nouveaux genres, dont l'autobiographie comme " a rejection of genre and a turn toward the real and the self, toward greater authenticity » (Beaty 8). L'Association, une coopérative d'artistes française fondée en 1990, fut la force motrice derrière cette transformation. L'Association a rompu avec le modèle corporatif industriel et du genre de la production de la BD et a repositionné la BD adulte comme une fonction du visuel plutôt que du littéraire. L'Association a ainsi pu faire émerger a latter day BD parallel to the historical development of the New Wave in cinema, which challenged the anonymous studio production through the cinema d'auteur in the same way that L'Association promoted the status of $\mathrm{BD}$ author as total creator, limited budget productions, and everyday subject matter. (Beaty 140-141)

Il y a des continuités évidentes entre la Nouvelle Vague et la NM : une prédilection pour l'errance, des récits épisodiques, souvent interrompus par des moments autoréflexifs et intertextuels (comme dans L'Épinard de Yukiko de Boilet), le brouillage de la distinction entre perception, pensée et imagination ( $A$ Patch of Dreams de Oda), un intérêt pour le décor ou son atmosphère du décor plutôt que pour le drame ou la psychologie des personnages (L'Homme qui marche de Taniguchi), le montage discontinu (les «coupes sèches» de Boilet), la dissociation de l'image et du son (Boilet), l'intérêt d'établir des relations entre les arts (Boilet), l'hybridité des genres ${ }^{6}$, l'amalgame des cultures "d'élite» et "populaire» (des BD traitant de thèmes existentiels, ex: Yume no akichi/Le Terrain vague de rêves de Oda Hideji, Keimusho no naka/Dans la Prison de Hanawa Kazuichi). La « Nouvelle Manga, » écrit Boilet dans son manifeste, « serait une initiative d'auteur (par opposition aux initiatives d'éditeur ou de libraire d'import, débouchant immanquablement sur des traductions - ou des importations — du tout-venant des séries à succès) » (Boilet). La NM reprend à son compte la notion d'auteur de la Nouvelle Vague, mais ré-imagine l'auteur comme une figure pleinement immergée dans les processus de la mondialisation

statiques et en coupes sèches. Laurence Grove affirme que malgré cet éloignement de la narration conventionnelle pour se rapprocher du visuel, la BD reste un médium hybride : une fois que le texte sera devenu complètement redondant, la $\mathrm{BD}$ aura évolué pour devenir quelque chose d'autre (Grove 55).

${ }^{6}$ Les fils de la Nouvelle Vague films mélangent différents genres: ex : thriller, BD, ciné-roman, journal, récit d'aventure, récit d'amour, poème en prose, publicité, journalisme, comédie musicale. 
plutôt qu'essayant furieusement de leur résister. $\mathrm{La}$ « $\mathrm{BD}$ d'auteur » (un terme que Boilet utilise comme synonyme de la NM) a le potentiel de devenir un art global indépendamment des contraintes géographiques et de genre dans la mesure où elle explore la vie quotidienne et reste libre des entraves commerciales. ${ }^{7}$ Paradoxalement, Boilet voit le manga comme une façon de « réeuropéaniser» la $\mathrm{BD}$ et par là-même de la «mondialiser». Ce faisant, le manifeste NM en rappelle un autre, qui préconise un art cinématographique mondial et indépendant, opposé à Hollywood : le fameux manifeste de Dogme.

À défaut d'être une tentative de faire revivre le cinéma national danois Dogme fut un moyen de remettre en question le cinéma commercial en repensant l'art du cinéma global de façon à défaire l'association trop étroite entre cinéma d'art et cinéma «national» (ex : films de patrimoine). En contrant les dynamiques du localisme alimentées par le globalisme, tout cela ayant poussé le mouvement des cinémas nationaux dans le local et même l'esprit de clocher, Dogme ramena l'attention sur l'art cinématographique de même que sur les conditions économiques et sociales de production. Si les films les plus connus de Dogme ont été réalisés par des cinéastes danois, le concept de Dogme a été adopté par un grand nombre de réalisateurs internationaux (même s'ils en ont décliné les règles de manière sélective). En encourageant des productions de films venant des "marges» (c'est-à-dire des petites nations), Dogme s'est présenté comme un contestataire de l'«illusionisme» à la Hollywood, son marketing et ses stratégies de distribution, ses budgets colossaux, ses célébrités, ses effets spéciaux, et ses conventions de genres. Influencé par le néo-réalisme italien, le Kino Pravda de Vertov et le cinéma-vérité, les réalisateurs de Dogme ont cherché à décomposer l'image, ignorant ou violant sans vergogne les règles de montage linéaire, jouant avec le visible et l'invisible, la dramatisation et la dédramatisation, etc. Dans Vow of Chastity, Lars von Trier fait écho à la critique de la Nouvelle Vague «d'une certaine tendance en cinéma », mais a aussi rejeté la notion «d'auteur » considéré comme le vestige du romantisme bourgeois : le « réalisateur » d'un film de Dogme ne doit pas être reconnu à cause de la nature collaborative de la réalisation d'un film. Cependant, outre l'interdiction de reconnaître l'auteur, le "Vow of Chastity » (où il est fait usage d'un équipement portable, d'une approche documentaire de la fiction, du tournage en plein air, d'un intérêt pour les tranches de vie plutôt que des histoires très dramatisées et

\footnotetext{
${ }^{7}$ Il n'en reste pas moins que même si les artistes de la NM s'expriment dans un style global de $\mathrm{BD} d^{\prime}$ auteur, par opposition au style commercial et à la $\mathrm{BD}$ traditionnelle créée pour un genre spécifique, plusieurs d'entre eux sont publiés non seulement dans des structures éditoriales alternatives comme Spore au Japon, Ego Comme X en France et Fanfare/Ponent Mon aux ÉtatsUnis, mais aussi dans les magazines à grand tirage: par exemple, Boilet a publié dans Big Comics au Japon.
} 
épiques) montraient clairement le désir d'authenticité et d'intimité de la Nouvelle Vague. Les réalisateurs de Dogme ont proclamé leur intolérance pour l'artifice et l'illusion, leur engagement à capturer "la vérité de la réalité », qui exige d'abandonner les avantages propres au cinéma d'Hollywood: éclairage, postproduction, accessoires, montage sonore, conventions de genre, intrigue linéaire, plans d'ensemble, plans d'analyse psychologique, prises de vue en studio, etc. ${ }^{8}$ Le manifeste de Boilet fait écho aux principes du manifeste de Dogme: le caractère universel de l'attrait d'une œuvre d'art se définit par le registre universel de la vie quotidienne (qu'elle soit autobiographique, documentaire ou fictive) plutôt que par son succès commercial. La distance séparant la BD commerciale du roman graphique d'auteur se révèle, en fin de compte, plus significative que celle entre les trois styles nationaux qu'on trouve dans les mangas, les comics, et la BD.

Certains critiques ont affirmé que, contrairement à la Nouvelle Vague, Dogme n'a pas été un mouvement spontané issu de conditions socioéconomiques particulières'. Le moment et l'endroit de l'apparition de Dogme n’ont pas été déterminés par un besoin manifeste : Lars von Trier en a proposé le concept comme moyen de subjuguer son propre perfectionnisme et ses difficultés à financer ses films; ce qui amène cette question: comment peut-on expliquer l'émergence de la NM? Celle-ci est-elle le résultat du désir de produire une nouvelle forme artistique ou s'agit-il plutôt d'une stratégie marketing? Derik Badman figure parmi les quelques critiques à avoir réfléchi à la motivation derrière ce que Boilet présente comme un nouveau genre, la NM. Selon Badman, Boilet « is attempting a marketing/terminological action to expose works that fall outside the stereotypical genres and could be enjoyed by the typical adult (or at least those outside the " otaku » or «BDphile» community)» (Kinderbook). Badman qualifie la tentative promotionnelle de «noble » en ce sens qu'elle a aidé à populariser les œuvres de mangakas du quotidien moins connues comme celles de Taniguchi, Tsuge and Nananan, tous trois ayant été rétroactivement associés avec la NM (Boilet les cite comme influences tous les trois dans son manifeste). En effet, le manifeste de Boilet s'organise clairement autour de la promotion et de la distribution. Par exemple, Boilet écrit que bien qu'un auteur comme

\footnotetext{
${ }^{8}$ Malgré les continuités évidentes entre la Nouvelle Vague française et Dogme (comme l'intérêt commun pour des récits libres, personnels, ambigus), on ne peut passer outre une différence majeure entre ces mouvements. La Nouvelle Vague promeut la notion d'auteur, dans le contexte de l'excellent cinéma de genre américain nouvellement reconnu, tandis que Dogme rejette autant l'auteur, reconnaissant à sa place la nature collective du tournage d'un film, que les contraintes de genre et la position dominante du cinéma américain dans me monde.

${ }^{9}$ Voir le livre de Jack Stevenson, Dogme Uncut: Lars von Trier, Thomas Vinterberg, and the Gang That Took on Hollywood.
} 
Mœbius soit connu au sein de l'industrie du manga et, à un moindre degré, par le grand public en général,

Cette reconnaissance n'est pas due aux ventes de [ses] ouvrages [...], mais à la seule promotion de [son] nom, une publicité orchestrée depuis la fin des années 80 par les éditeurs, la presse, les libraires et les institutions françaises. Les BD de [cet auteur] n'ont pourtant que peu de chance de toucher un large public au Japon, ce large éventail de lecteurs que j'évoquais précédemment... [parce que] Comme souvent avec la SF, les histoires de Mœbius sont très connotées, il faut une culture et une nostalgie particulières pour les apprécier, à la fois une culture de la $\mathrm{BD}$, une culture «de Mœbius », mais aussi une nostalgie de la BD de la fin des années 70, en tous cas une culture et une nostalgie que la plupart des lecteurs japonais n'ont pas. (Boilet)

De plus, Boilet affirme que l'émergence et le succès de la Nouvelle BD, au début des années 1990, grâce à des éditeurs comme l'Association et Ego Comme X, était dû précisément à la transformation de la $\mathrm{BD}$ des années 80 , commerciale et figée dans certains genres, en une BD faite de récits de la vie quotidienne qui plaisait à un nouveau lectorat, dépassant ainsi le marché étroit des fans de BD. Alors que les œuvres des auteurs de la Nouvelle BD étaient traduites pour les marchés d'autres pays européens et pour les États-Unis, les $\mathrm{BD}$ commerciales sont restées limitées au marché franco-belge.

S’appuyant sur une stratégie de marketing déjà employée, habituellement réservée aux genres cinématographiques - l'hybridation de deux genres pour attirer un public plus large — mais en l'appliquant à un média différent (la BD), Boilet cherche à légitimer la NM par le biais de son association avec le cinéma, particulièrement la Nouvelle Vague, avec laquelle la NM partage plus de similarités qu'elle n'en a avec la BD traditionnelle ou le manga grand public. Boilet admet être influencé par des mangakas comme Yoshiharu Tsuge, Naito Yamada, Kiriko Nananan, dont les mangas d'auteur s'apparentent davantage aux films de Yasujirô Ozu et de Jacques Doillon qu'aux BD commerciales ou aux films de Luc Besson. En racontant des histoires de la vie quotidienne, affirme Boilet, la BD devient plus universelle et, du point de vue du lecteur étranger, plus « française », car elle fait partie d'une culture française plus large, incluant le cinéma français. En effet, le succès que Boilet présage pour la NM se base sur le fait que la commercialisation du cinéma français fonctionne au Japon, le second plus gros marché dans le monde pour le cinéma français.

Étudions maintenant certains exemples de NM. L'œuvre inaugurale de la NM, L'Épinard de Yukiko de Boilet, une manga autobiographique relatant l'idylle entre Boilet, un mangaka français habitant au Japon, et Yukiko, une Japonaise. Boilet adapte pour le médium de la BD de nombreuses techniques qui ont fait de la Nouvelle Vague un cinéma unique. L'Épinard de Yukiko s'ouvre sur une série de pages, consistant en trois « bandes » correspondant à trois points 
de vue légèrement différents de la même scène de rue : la variation de l'angle et/ou de l'échelle du plan est mineure et inutile pour raconter une histoire, c'està-dire qu'on a une version BD du «jump cut» tel qu'utilisé, par exemple, par Godard dans $\grave{A}$ Bout de souffle. Les pages suivantes sont remplies de dessins de Yukiko qui se répètent de façon obsessionnelle, encore une fois selon des points de vue différents et à des distances différentes, et qui ne font pas partie de l'histoire : aucun de ces dessins n'est immédiatement ou définitivement identifiable comme représentant un moment particulier dans une narration se développant chronologiquement. Il faut plutôt lire les événements que ces dessins représentent comme ayant lieu dans le présent et, en même temps, comme manifestations du rêve, des souvenirs ou de l'imagination de l'auteurpersonnage. La scène du dialogue entre Boilet et Yukiko dans le restaurant digresse constamment vers des associations libres de pensées et de souvenirs tandis que, paradoxalement, cette scène s'attache à enregistrer de manière minutieuse le moindre geste mineur de la femme dont Boilet est amoureux. «L'histoire » de leur relation vouée à l'échec est rapportée non pas au moyen d'une série d'événements chronologiques, mais au moyen d'actions étalées dans le temps en transcrivant d'une manière à la fois distraite et méticuleuse les mouvements et les gestes, aussi petits et insignifiants soient-ils. À la manière de Godard, Boilet juxtapose, au hasard, des dessins de Yukiko au présent, avec des dessins d'elle comme elle existerait dans son imagination et des dessins de parties d'elle tirées d'abord de son carnet d'esquisses puis de son ordinateur alors qu'il édite la BD dans laquelle elle est un personnage.

La méthode photo-réaliste de Boilet consiste à photographier ses sujets et ses paysages, puis à utiliser des filtres pour transformer les photos en dessins formés de traits; il inclut aussi quelques dessins aux traits traditionnels dans le livre $^{10}$. L'Épinard de Yukiko présente un certain nombre d'éléments caractéristiques de la Nouvelle Vague, telles que l'autoréflexion, la fragmentation, la stylisation, l'organisation en épisodes et l'intertextualité. Ss sur le site boilet.net, Jérôme Attal rapproche d'ailleurs Yukiko et cinéma: « c'est à la fois un tableau et un court métrage de cinéma, étendus sur plusieurs planches de bd ", tandis que d'autres commentateurs refusent de l'appeler manga précisément à cause de sa nature exclusivement visuelle, affirmant que " it is

${ }^{10}$ Certains critiques, qui ne portent pas attention à l'utilisation que fait Boilet des photos modifiées, affirment être en mesure de détecter la touche véritable de l'artiste dans l'œuvre, par opposition à un simple filtre photoshop (voir notamment la critique de Jonathan Ellis). D'autres critiquent l'affection de Boilet pour les techniques de montage au détriment de la narration, et remarquent de manière sarcastique qu'on pourrait aimer le livre pour la seule raison qu'il regorge de jolies photos d'une fille japonaise (voir la critique de Joanna). Kelly Sue DeConnick trouve les personnages "woefully underdeveloped", mais l'art "stunningly beautiful". 
mainly devoted to silent actions, and there is hardly any speech. » (Slightly Biased Manga). Dans Libération, Éric Loret loue l'intimité à laquelle parvient Boilet au moyen «d'une caméra subjective » ce qui appelle une comparaison directe avec le cinéma français : "Yukiko dans les mains de Boilet est la plus belle fille au monde, belle comme Anna Karina à travers le regard de Godard, belle comme Maggie Cheung examinée de près par Assayas» («Baka updates»). Robert Sparing loue la manipulation filmique que Boilet fait du temps, « shifting scenes back and forth to the beginning of the relationship and the end, placing dreamy imagery intermittent with the more linear panels. » Boilet utilise des logiciels de dessin pour atteindre un certain photoréalisme stylisé rappelant la fusion du réalisme documentaire et de la stylisation caractéristique de la Nouvelle Vague : «In combining the two almost seamlessly, he [Boilet] creates a photorealism that is, to a small degree, attempting not to be realistic. » (Sparling). Boilet sépare souvent l'image du son, réalisant ainsi un effet qui rappelle les films de Godard : "Sounds plays a role in a scene (page 31) where a repeated pair of sentences is at first shown with ellipses and only a word or two, but, as Boilet moves forward towards the sound, more words are shown until the complete sentences can be made out. » (Badman)

Non seulement Boilet compose les cases de manière à ce que l'on voie à travers ses yeux (ceux de l'auteur-personnage), mais il lui arrive de varier souvent l'agencement de la focalisation pour créer l'impression que les yeux du personnage errent ici et là. Le regard selon la perspective de la première personne se voit complexifié par le regard artistique, produisant un effet autoréflexif et métafictionnel :

Throughout the book we see full page excerpts from Boilet's daily calendar where he writes notes and sketches. These pages not only serve as date markers for events in the book, they also add a layer of the story of the story. They create the illusion...of life being represented by art. [...] A sketch of a hand in the date book on page 12 becomes Yukiko's hand on her collar on page 13. The notes precede the comic, yet the story precedes the notes. [...] Towards the middle of the book, Boilet asks Yukiko about making a comic about her, and then a few pages later he is working on an earlier part of the story on his computer when she calls him. A circular movement is created by this looking back upon an earlier page of the book. (Badman) ${ }^{11}$

\footnotetext{
11 Badman observe fort justement qu'en contraste avec l'expérimentation visuelle invoquée comme critère de la NM dans le manifeste de Boilet, la mise en page ici est « almost all regular: three, four, or six identical panels per page. There are the occasional variations but always with rectangular panels and no bleeds. [...] On two occasions he overlaps panels, both are at the conclusion of sex scenes, an effective place to have larger panels breaking out of bounds. ». Ironiquement, ce qui impressionne Badman à propos de l'œuvre de Boilet, ce sont ses aspects formels (principalement la perspective à la première personne) plutôt que le récit ou les personnages, précisément les deux aspects que la NM est supposée mettre en valeur.
} 
Boilet a travaillé avec la mangaka Kan Takahama sur une sorte de suite, Mariko Parade, qui contient aussi les éléments de la Nouvelle Vague mentionnés cidessus, mais encore plus autoréflexifs. Takahama a écrit et dessiné un cadre narratif englobant une série de pièces courtes que Boilet voulait rassembler et dont l'unique point commun réside dans l'usage de son modèle, Mariko. La contribution de Takahama change tout le contexte des séquences de Boilet: L'Épinard de Yukiko est à propos de Yukiko/Mariko. Mariko Parade parle de la fascination de Boilet pour Yukiko/Mariko (Garrity). La plupart des critiques s'accordent à dire, cependant, que les pièces courtes de Boilet ne s'intègrent pas complètement au reste de l'histoire.

Récit autobiographique de Kazuichi Hanawa, Dans une prison raconte les trois années que l'auteur a passées dans le système pénitentiaire japonais. Comme l'œuvre de Boilet, Dans une prison qui est composé de dessins à l'encre noire ou sépia, remplis de listes décrivant l'emploi du temps et de pages illustrant des repas, ressemble à une collection de vignettes plus qu'à une narration cohérente. Au lieu de construire une histoire, Hanawa passe d'un sujet à un autre, d'un jour à l'autre, créant la sensation d'un laps de temps sans fin ni forme. De petits événements, comme un item nouveau au menu du dîner, prennent une énorme signification et sont narrés avec des détails minutieux (Garrity). Dans l'adaptation filmique à la manière d'Ozu, qui polarise l'attention sur la cellule que Hanawa partage avec quatre autres criminels, le réalisateur coréo-japonais Sai explore d'une manière tout aussi détaillée les règlements et routines quotidiennes de la vie en prison : repas, lessive, nettoyage, bains, exercices, fabrication de contenants pour boittes de papier mouchoir à l'atelier.

Évoquant l'atmosphère de résignation qui nous est familière dans les films de Ozu, et aussi nous rappelant le film Ikiru [Vivre] (1952) de Kurosawa - l'histoire d'un bureaucrate apathique qui subit une transformation spirituelle après avoir appris qu'il va mourir d'un cancer — le manga Aruku Hito/L'Homme qui marche/The Walking Man, Jiro Taniguchi incarne les préceptes de la NM. En effet, L'Homme qui marche s'empare des activités mineures de la vie quotidienne pour les dessiner dans un style graphique très détaillé plus communément associé à la BD européenne (Arnold). Comme Ozu, Taniguchi utilise des allusions associatives habituellement tirées de la nature et réaffirme sa vision d'un temps cyclique, mythique ou transcendantal, évoquant l'intemporalité et l'indifférence de la nature ainsi que l'insignifiance des êtres humains (Desser $19)^{12}$. Il n'y a pas d'intrigue : le manga dépeint simplement un salarié anonyme

12 Par deux fois dans la trame de Ikiru le protagoniste chante la fameuse chanson Gondola Song de 1915 qui résume à merveille l'attitude mono no aware d'Ozu (la conscience du caractère transitoire de toutes choses): Life is brief // fall in love, maidens // before the crimson bloom 
lors de différentes promenades à la ville et à la campagne. Plutôt que de construire un drame à partir d'événements significatifs, Taniguchi — comme $\mathrm{Ozu}$ - s'attarde sur les petits moments de la vie d'un homme. Chaque chapitre présente une promenade à un endroit différent, " with emphasis placed on the weather, the changes of season, encounters with animals and brief, mostly silent exchanges with people» (Arnold). Les références subtiles de Taniguchi à la nature fonctionnent d'une manière similaire au célèbre "pillow shots» de Ozu, des scènes de la nature - scènes extrêmement longues ou gros plans de champs, de la mer, d'un jardin, de la cime des arbres, etc. — intercalées entre des épisodes qui forment le récit du film. Le but de ces "pillow shots » est de laisser le spectateur se reposer et réfléchir à ce qui s'est passé, mais aussi de placer des événements humains dans le contexte plus large des forces naturelles, et de présenter l'existence humaine comme faisant partie du cycle de la nature, sur lequel nous n'avons aucun contrôle. L'Homme qui marche devient spécialement cinématique quand ses scènes ont lieu en imitation du plan contre plan filmique, quand une case montrant un homme qui regarde au loin est suivie d'une case montrant l'objet qu'il regarde. Les pages s'organisent selon un "style rhétorique »: « tall panels are used for trees, wide panels for scenic vistas, smaller wider panels for the man's head looking off panel at something, single panel pages for slowed down moments » (The Walking Man).

Puisque Boilet établit la légitimité de la NM comme genre nouveau à partir de son association avec le cinéma, on peut se demander si la NM serait "plus cinématique » que le manga ou la $\mathrm{BD}$. En premier lieu, même la BD traditionnelle emprunte une panoplie de techniques au cinéma: plan général/panorama; plan moyen, plan américain, plan rapproché, gros plan, plongée, contre-plongée, travelling ${ }^{13}$. En second lieu, les critiques distinguent souvent le manga de la BD traditionnelle, précisément en faisant valoir la nature cinématique du manga, que les critiques font remonter à après la Seconde Guerre mondiale quand Osamu Tezuka

helped revolutionize the art of comics in Japan by decompressing story lines... drawing novelistic manga that were hundred, even thousands of pages long, and incorporat[ing] a lot of cinematic techniques (camera angles, creating a sense of movement via the page layouts). [...] The result was a form of comics with far fewer words than American or European comics...that uses far more frames and pages to depict an action or a thought. (Schodt 25)

// fades from your lips // before the tides of passion // cool within you, // for those of you // who know no tomorrow.

${ }^{13}$ Le cinéma et la $\mathrm{BD}$ ont aussi une parenté historique: l'âge d'or du cinéma américain, par exemple, les années 1930 et 1940, correspond à l'âge d'or des comics de superhéros. Autant le cinéma d'après-guerre que la $\mathrm{BD}$ ont fait l'objet d'expérimentations similaires à partir des conventions établies. 
Schodt attribue justement la manière particulière de raconter caractéristique des mangas au style cinématique du manga :

The cinematic style enables manga artists to develop their story lines and characters with more complexity and psychological and emotional depth. Like good film directors, they can focus reader attention on the minutia of daily life-on scenes of leaves falling from a tree, or steam rising from a bowl of noodles, or even the pregnant pauses in a conversation-and evoke memories and associations that are deeply moving. (Schodt 26)

Le génie cinématique du mangaka apparaît évident dans sa mise en page magistrale :

They have learned to make the page flow; to build tension and to invoke moods by varying the number of frames used to depict a sequence; to use the cinematic techniques of fade out, fade in, montage and even superimposition. Like Japanese poetry, Japanese comics tend to value the unstated; in many cases, the picture alone carries the story. (Schodt 20-21)

Le lecteur de manga « parcourt » plutôt qu'il ne « lit» un manga « in contrast to the American comic, which is read slowly to savor lavishly detailed pictures and to absorb a great deal of printed information » (Schodt 23).

D'autre part, certains critiques prétendent qu'il serait insensé de parler d'un style "cinématographique» en ce qui concerne les comics. Selon Christiansen, "What in comics is called the cinematographic style is the particular dynamisation of the graphic language...using graphic equivalents to those narrative devices, e.g. close up, chiaroscuro, point of view and dynamic editing of camera-angles »(107-108). Christiansen affirme cependant que les procédés cinématographiques dans les comics ne peuvent pas être considérés comme du «style cinématique » parce qu'ils doivent nécessairement se plier aux contraintes de chaque médium. Ainsi, si le cinéma (et il veut dire le cinéma classique de montage linéaire) cache le travail de production et permet aux spectateurs d'entrer dans la diégèse et de s'identifier étroitement avec les personnages et l'action. En revanche, le lecteur de comics n'est jamais vraiment immergé dans l'œuvre. La transparence filmique dépend de deux caractéristiques du médium cinématographique qu'on ne trouve pas dans le médium comics: le mouvement et l'aspect déictique. En revanche, les comics sont constitués d'images fixes et "foreground the presence of enunciator [the narrator's construction of the diegesis or the artist's pencil] which blocks the identification process in making it more difficult for the spectator to create the illusion of being...the unique origin of all identification »(Christiansen 115). Puisque l'identification n'est pas aussi étroite, les éléments qui rattachent le spectateur à la narration visuelle — procédés dits de « eye-line match» — ne sont pas essentiels 
dans les comics, qui se caractérisent généralement par des coupures elliptiques (sauts temporels) entre des images successives qui sont plus discordantes au cinéma (Christiansen 117), (coupures qui sont justement utilisées par les réalisateurs de la Nouvelle Vague pour cette raison).

Cet argument s'applique-t-il aussi à la NM? Évidemment, la NM se compose aussi d'images fixes et n'a pas l'aspect déictique d'une image photographique d'une image photographique (même si cela se complexifie dans le cas de la méthode photo-réaliste de Boilet). Il est indiscutable cependant qu'il existe une continuité entre, d'une part, la NM de Boilet, de Taniguchi, d'Hanawa ou d'Oda et, d'autre part, les adaptations en film de la NM. Par exemple, Man without Talent (1991), tiré du manga autobiographique de Tsuge Yoshiharu, présente un mangaka dans la misère qui lance un commerce de belles pierres dans une cabane près de la rivière. Le film, comme le manga, met l'emphase sur une narration au rythme visuel lent plutôt que de braquer les projecteurs sur de grands événements dramatiques. Blue (2001), tiré du manga de Kiriko Nananan, raconte comment une écolière introvertie tombe amoureuse d'une de ses camarades de classe et commence avec elle une idylle. Ce film aussi se distingue par un rythme calculé, un ton calme et des prises longues et ininterrompues qui le distinguent des films plus conventionnels. Strawberry Shortcakes (2006), tiré d'un autre manga de Kiriko Nananan, raconte l'histoire de quatre filles vivant à Tokyo. La présentation non-linéaire des histoires de ces femmes renforce la connexion entre les personnages, ce qui crée une résonance entre leur vie, leurs sentiments et leurs déceptions. Comme le manga, le film présente un caractère épisodique en passant d'un personnage au suivant sans trop pousser le développement de leur situation.

Il faut se rappeler que le dessin dans les BD reste fondamentalement un processus narratif. Ainsi, la tendance à s'éloigner du littéraire dans la BD pour se rapprocher des arts visuels (en particulier, le cinéma) doit aussi être imputée aux bédéistes qui choisissent d'harmoniser leur esthétique visuelle avec des façons de raconter nouvelles et innovantes (Beaty 248). La tendance vers le visuel n'est pas simplement un rejet de l'aspect littéraire ou textuel de la BD, mais plutôt un réajustement à de nouvelles façons de raconter non-séquentielles (comme celles de la Nouvelle Vague). C'est la seule manière d'expliquer que la NM, paradoxalement, préfère la narration à l'illustration dans un contexte où la critique a mis en lumière une tendance vers le visuel dans les BD depuis le début des années 1990. Qualifier la NM de « cinématique », dès lors, ce n'est pas juste faire référence aux techniques visuelles particulières que nous associons avec le cinéma, mais c'est aussi faire référence à une façon particulière de raconter propre à la Nouvelle Vague et au cinéma japonais. Il y a d'indéniables similarités 
entre les caractéristiques de la Nouvelle Vague comme l'errance, l'organisation en épisodes, des récits dédramatisés structurés autour de séries de moments librement connectés, des moments passagers et ce que David Desser appelle, dans Eros plus Massacre, "le paradigme classique » du cinéma japonais, à distinguer du paradigme moderne des humanistes d'après-guerre représenté par Kurosawa, et du paradigme moderniste, exemplifié par la nouvelle vague japonaise. La narration classique est « chronological, episodic, cyclical, mythic, transcendental, » la narration moderne, « chronological, causal, linear, historical, individual » et la narration moderniste, " achronological, arbitrarily episodic, acausal, dialectical, anti-mythic and anti-psychological, metahistorical » (Desser 17). On trouve le meilleur exemple de paradigme classique (qui ne se limite pas à une période historique particulière) dans les films de Ozu dont Desser compare le mode narratif avec le kabuki et le roman japonais. Les films de Ozu perturbent la linéarité narrative, accentuent les manipulations spatiales, s'appuient sur des ellipses temporelles, emploient une structure épisodique, évitent les moments culminants pour explorer le banal et la stérilité événementielle de la vie quotidienne (Desser 17-18). Toutes ces stratégies provoquent chez le spectateur (et chez le lecteur dans un manga de ce genre) la réponse spécifiquement japonaise du "mono no aware», une compréhension intuitive du passage du temps et de son inéluctabilité (cela est particulièrement vrai des œuvres de Taniguchi et Hanawa). Boilet imagine la Nouvelle Manga comme utilisant des histoires quotidiennes inspirées des mangas japonais afin de compenser l'emphase excessive sur l'illustration qu'il perçoit dans la BD française. Paradoxalement, l'incorporation des histoires quotidiennes ne résulte pas en une emphase plus grande sur l'histoire; mais à l'opposé : la narration libre et épisodique de la Nouvelle Manga ou le manque de narrativité a servi à recentrer notre attention sur le plan visuel. 


\section{Bibliographie}

Arnold, A. « Manga Mon Amour. » «Manga My Love. » Time. Nov. 11, 2004, http://www.time.com/time/columnist/arnold/article/0,9565,781511,00.html. Consulté le 17 février 2012.

Badman, D., 2005. Kinderbook and Mariko Parade, http://madinkbeard.com/archives/kinderbook-and-mariko-parade. Consulté le 17 février 2012.

. The Walking Man.

http://www.comicbookgalaxy.com/review_101705_walkingman_DB.html. Consulté le 17 février 2012.

. Yukiko's Spinach. http://madinkbeard.com/archives/yukikos-spinach-byboilet. Consulté le 17 février 2012.

Baka-Updates. http://www.mangaupdates.com/series.html?id=33706. Consulté le 17 février 2012,

Beaty, B. Unpopular Culture: Transforming the European Comic Book in the 1990s. University of Toronto Press, 2007.

Bilal, E. The Carnival of Immortals. Les Humanoïdes Associés, 1980.

Boilet, F. Nouvelle Manga Manifesto. 2001. http://www.boilet.net/am/nouvellemanga_manifeste_1.html, Consulté le 17 février 2012.

Carrier, D. The Aesthetics of Comics. Pennsylvania State University Press, 2000.

Charlier, J-M. and Uderzo, A., Tanguy et Laverdure. The Adventures of Tanguy and Laverdure. Editions Dargaud. 1959-1971.

Christiansen, H-C. «Comics and film: a narrative perspective », in Comics and Culture: Analytical and Theoretical Approaches to Comics. Magnussen, A. and Christiansen, H-C. ed. Museum Tusculanum Press. 2000. pp. 107-121.

Connie, Slightly Biased Manga: Manga Only If I Like It. http://slightlybiasedmanga.com/category/series/yukikos-spinach/. Consulté le 17 février 2012.

DeConnick, K.S. Yukiko's Spinach. http://www.artbomb.net/detail.jsp?tid=442. Consulté le 17 février 2012.

Desser, D. Eros plus Massacre: An Introduction to the Japanese New Wave Cinema. Indiana University Press, 1988.

Ellis, J. Review: Yukiko's Spinach. http://www.popimage.com/content/viewnews.cgi?newsid1077089884,3 9827. Consulté le 17 février 2012.

Francq, P. and Hamme, J. Largo Winch. Dupuis. 20 albums. 1990 à 2015. 
Garrity, S. K. Doing Time. http://shaenon.livejournal.com/38670.html\#cutid1. Consulté le 17 février 2012.

Garrity, S. K. Mariko Parade. http://shaenon.livejournal.com/41787.html. Consulté le 17 février 2012.

Grove, L. Comics in French: The European Bande dessinee in Context. Berghahn Books, 2010.

Hatfield, C. An art of tensions. In A Comics Studies Reader. Heer, J. and Worcester, K. ed. University Press of Mississippi, 2009. pp. 132-148.

Johanna. "Yukiko’s Spinach » in Manga Reviews, February 12, 2006. http://comicsworthreading.com/2006/02/12/yukikos-spinach/. Consulté le 17 février 2012.

Mœbius. Blueberry. Dargaud, 1963.

Schodt, F. Dreamland Japan: Writings on Modern Manga. Stone Bridge Press, 1996. . Manga! Manga! The World of Japanese Comics. Kodansha International, 1983.

Sparling, R. Yukiko's Spinach. http://www.fanboyplanet.com/comics/rsyukikospinach.php. Consulté le 17 février 2012.

Stevenson, J., Dogme Uncut: Lars von Trier, Thomas Vinterberg, and the Gang That Took on Hollywood. Santa Monica Press, 2003. 